\title{
General Relativistic Simulations of Stellar Core Collapse and Postbounce Evolution with Boltzmann Neutrino Transport
}

\author{
M. Liebendörfer*, O. E. B. Messer*, A. Mezzacappa ${ }^{\dagger}$ and W. R. Hix* \\ *Department of Physics and Astronomy, University of Tennessee, Knoxville, TN-37996 \\ and Physics Division, Oak Ridge National Laboratory, Oak Ridge, TN-37831 \\ ${ }^{\dagger}$ Physics Division, Oak Ridge National Laboratory, Oak Ridge, TN-37831
}

\begin{abstract}
We present self-consistent general relativistic simulations of stellar core collapse, bounce, and postbounce evolution for 13, 15, and 20 solar mass progenitors in spherical symmetry. Our simulations implement three-flavor Boltzmann neutrino transport and standard nuclear physics. The results are compared to our corresponding simulations with Newtonian hydrodynamics and $\mathrm{O}(\mathrm{v} / \mathrm{c})$ Boltzmann transport.
\end{abstract}

\section{INTRODUCTION}

A supernova explosion is a dramatic event that includes such a rich diversity of physics that, with current computer hardware, a self-consistent model based on numerical simulations can not possibly include all of them at once. After stellar core collapse, a compact object at the center of the event is formed, requiring a description in general relativity. Neutrinos radiating from this central object are strongly coupled to the matter at high densities before streaming out at lower densities. Multi-frequency radiation hydrodynamics must be used to quantify the energy that these neutrinos deposit in the material behind the shock. Moreover, evidence suggests that this heating drives convection behind the shock, and significant rotation and strong magnetic fields might also be present. Observations of neutron star kicks, mixing of species, inhomogeneous ejecta, and polarization of spectra support the presence of asymmetries in supernova explosions (e.g. Tueller et al. [1], Strom et al. [2], Galama et al. [3], Leonard et al. [4], and references therein). Motivated by such observations, various multi-dimensional explosion mechanisms have been explored (Herant et al.[5], Miller et al.[6], Herant et al.[7], Burrows et al.[8], Janka and Müller [9], Mezzacappa et al.[10], Fryer [11], Fryer and Heger [12]) and jet-based explosion scenarios have recently received new momentum (Höflich et al. [13], Khokhlov et al. [14], MacFadyen and Woosley [15], Wheeler et al. [16]). Many exciting aspects of jets are discussed in these proceedings. However, for the time being, one has to single out a subset of the known physics and to investigate the role each part plays in a restricted simulation. It is natural to start with ingredients that have long been believed to be essential for the explosion and to add modifiers in a systematic way until the observables can be reproduced (see e.g. Mezzacappa et al. [17]). Spherically symmetric supernova modeling has a long tradition and is nearing a definitive point in the 
sense that high resolution hydrodynamics, general relativity, complete Boltzmann neutrino transport, and reasonable nuclear and weak interaction physics are being combined to dispel remaining uncertainties.

Our simulations are based on the three-flavor Boltzmann solver of Mezzacappa and Bruenn $[18,19,20]$ that was consistently coupled to hydrodynamics on an adaptive grid and extended to general relativistic flows (Liebendörfer [21]). We performed relativistic simulations for progenitors with different masses and compare the results to the counterpart simulations in Newtonian gravity with $\mathrm{O}(\mathrm{v} / \mathrm{c})$ Boltzmann transport (Messer [22]). While the quantitative results are sensitive to the inclusion of all neutrino flavors and general relativity (Bruenn et al. [23]), we find the same qualitative outcome as did Rampp and Janka [24] in their independent simulations with one-flavor O(v/c) Boltzmann transport: Given current input physics, there are no explosions in spherical symmetry without invoking multidimensional effects (Mezzacappa et al. [17], Liebendörfer et al. [25]). This is consistent with the results of Wilson and Mayle [26], who found explosions only by including neutron finger convection.

\section{CORE COLLAPSE AND NEUTRINO BURST}

The simulations discussed herein are initiated from progenitors with main sequence masses of $13 \mathrm{M}_{\odot}, 20 \mathrm{M}_{\odot}$ (Nomoto and Hashimoto [27]), and $15 \mathrm{M}_{\odot}$ (Woosley and Weaver [28]). We use in our models the equation of state of Lattimer and Swesty [29] and "standard" weak interactions (e.g. Bruenn [30]). The first phase in the simulations leads through core collapse to bounce and shock formation. When the shock passes the neutrinospheres approximatively $4 \mathrm{~ms}$ after bounce, an energetic neutrino burst is released from the hot shocked material, rendering it "neutrino-visible" to the outside world. The corresponding deleptonization behind the shock is dramatic. The energy carried off with the neutrino burst enervates the shock in both the NR and the GR cases. A pure accretion shock continues to propagate outwards as infalling material is dissociated and layered on the hot mantle. This stage is the definitive end of a "prompt", i.e. purely hydrodynamic, explosion.

The neutrino luminosities from the general relativistic simulations are shown in Fig. (1). The electron neutrino luminosity slowly rises during collapse and decreases as the core reaches maximal density. It remains suppressed for the $\sim 4$ ms the shock needs to propagate to the electron neutrinosphere. The most prominent feature is the electron neutrino burst, reaching $3.5 \times 10^{53} \mathrm{erg} / \mathrm{s}$ at shock breakout, and declining afterwards.

The initial similarities in density and temperature in the inner parts of the three cores are responsible for the similar evolution of each core with respect to collapse, bounce, and the signature of the electron neutrino burst (Messer [22]). This is shown in Figs. (1) and (2). Any initial difference in the electron fraction, $Y_{e}$, in the inner part of each core cannot survive in regions that reach sufficient densities for neutrino trapping and equilibration to occur. If a mass element reaches neutrino trapping density, the total lepton fraction, $Y_{l}$, becomes the variable determining the state of the mass element. At equilibrium, the electron capture and neutrino absorption rates are related to one another through detailed balance, and the final $Y_{e}$ becomes a function of the local temperature 

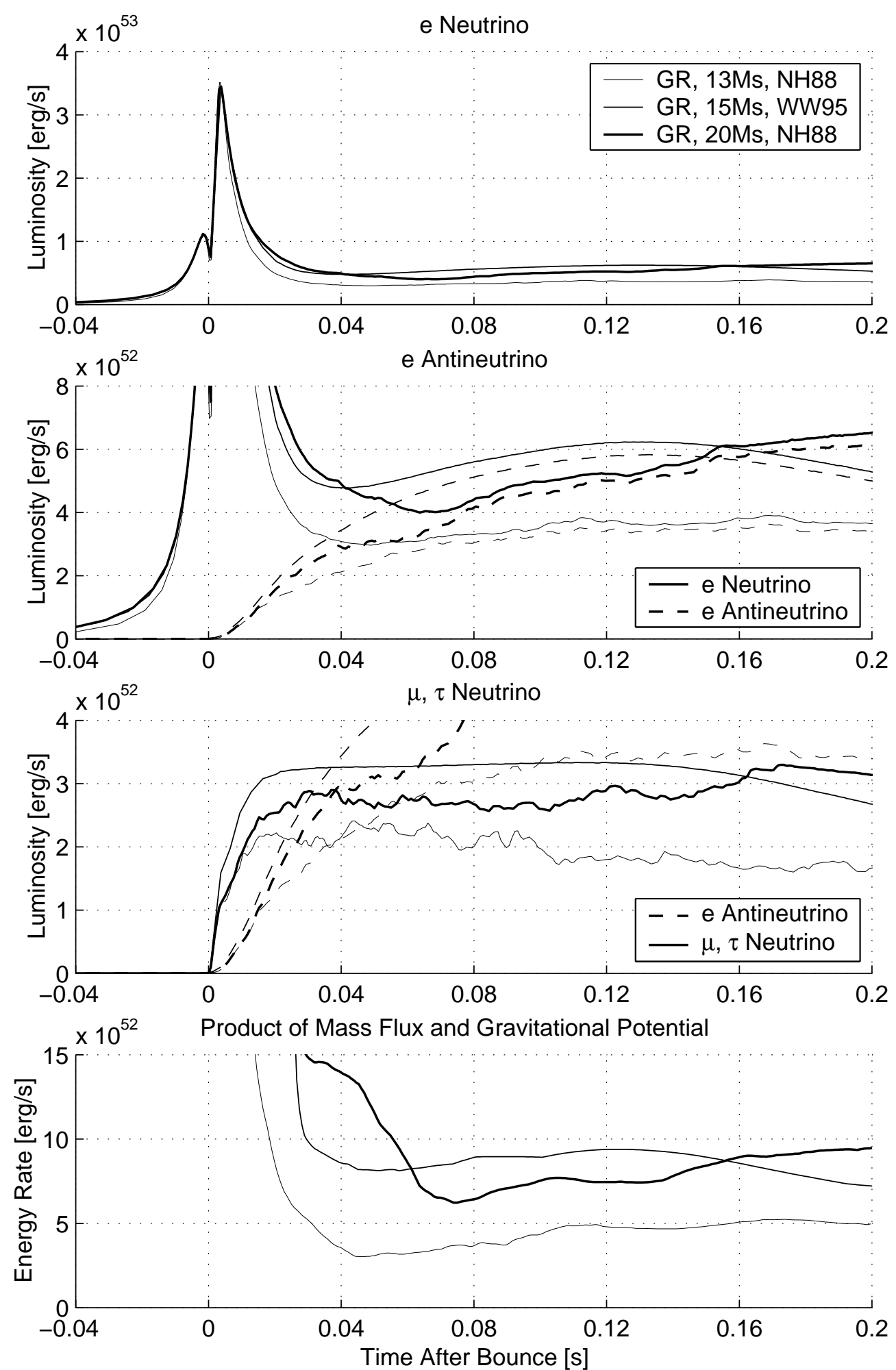

FIGURE 1. Luminosities for all flavors in the GR simulations. The last graph shows an energy rate calculated by the product of the gravitational potential at the neutrinosphere and the mass flux crossing it. 

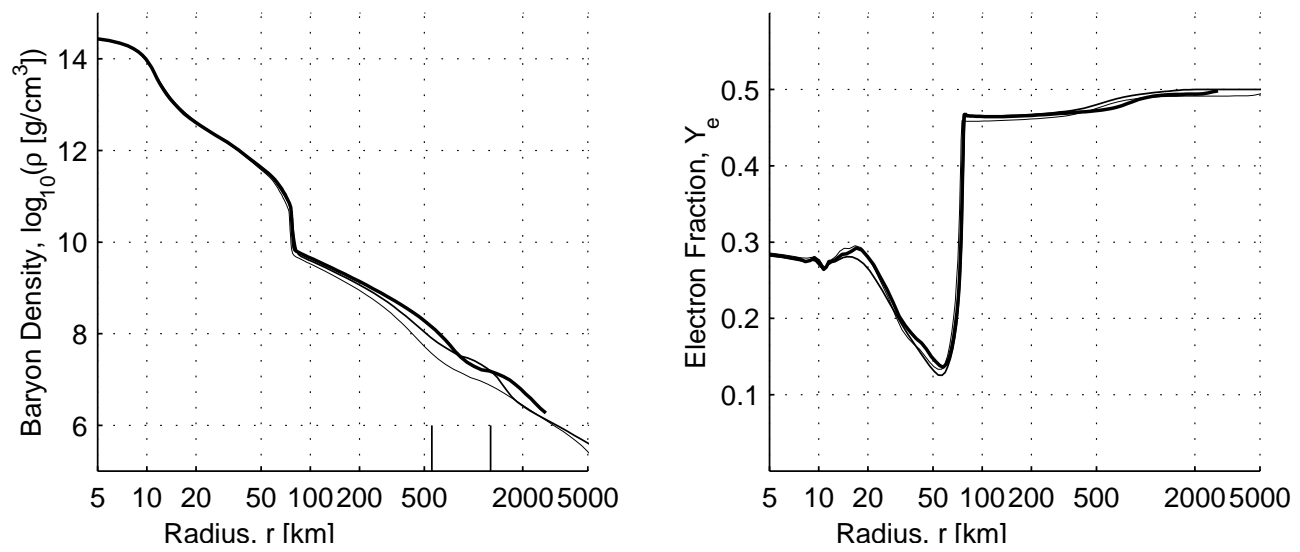

FIGURE 2. Similar core profiles in the GR cases at $10 \mathrm{~ms}$ after bounce.

and density through the electron chemical potential. Since the inner temperature and density profiles differ very little between the models, each settles to essentially the same equilibrium $Y_{e}$ deep in the core. This determines the size of the homologous inner core and, consequently, the position of shock formation. Since this position sets the amount of mass that has to be dissociated when the shock ploughs to the neutrinospheres, it strongly influences the shock energy. Thus, a strikingly similar shock initiation is found for the three progenitors. However, the initially dissociated mass differs largely between the GR and NR cases because of the smaller enclosed mass at shock formation due to the GR effects in the gravitational potential (GR: $0.53 \mathrm{M}_{\odot}, \mathrm{NR}: 0.65 \mathrm{M}_{\odot}$ ). The luminosities in the GR and NR cases are very similar for the electron neutrino burst. Afterwards, the GR luminosities are generally 10\% - 20\% larger than the NR luminosities.

\section{HEATING AND ACCRETION}

In the standard picture, the ensuing evolution is driven by electron neutrino heating. Electron flavor neutrinos diffuse out of the cold unshocked core and are created in the accreting and compactifying matter around the neutrinospheres in a hot shocked mantle. If the heating is not sufficient to revive the shock within the first $\sim 200 \mathrm{~ms}$ after bounce (as is the case in our simulations), the material in the heating region is drained from below (Janka [31]), and the conditions for heating deteriorate because of the shortened time the infalling matter spends in the heating region. We observe this process earlier in the general relativistic simulations than in the nonrelativistic simulations.

The shock trajectories for the different progenitors are shown in Fig. (3). The main difference between the GR and NR simulations stems from the difference in the size of the proto-neutron star, which is caused by the nonlinear GR effects at very high densities in the center of the star. At radii of order $100 \mathrm{~km}$ and larger, the gravitational potential becomes comparable in the GR and NR cases. However, large differences arise if the steep gravitational well is probed at different positions in the GR and NR simulations, as happens with accretion down to the neutrinospheres. The deeper neutrinospheres 


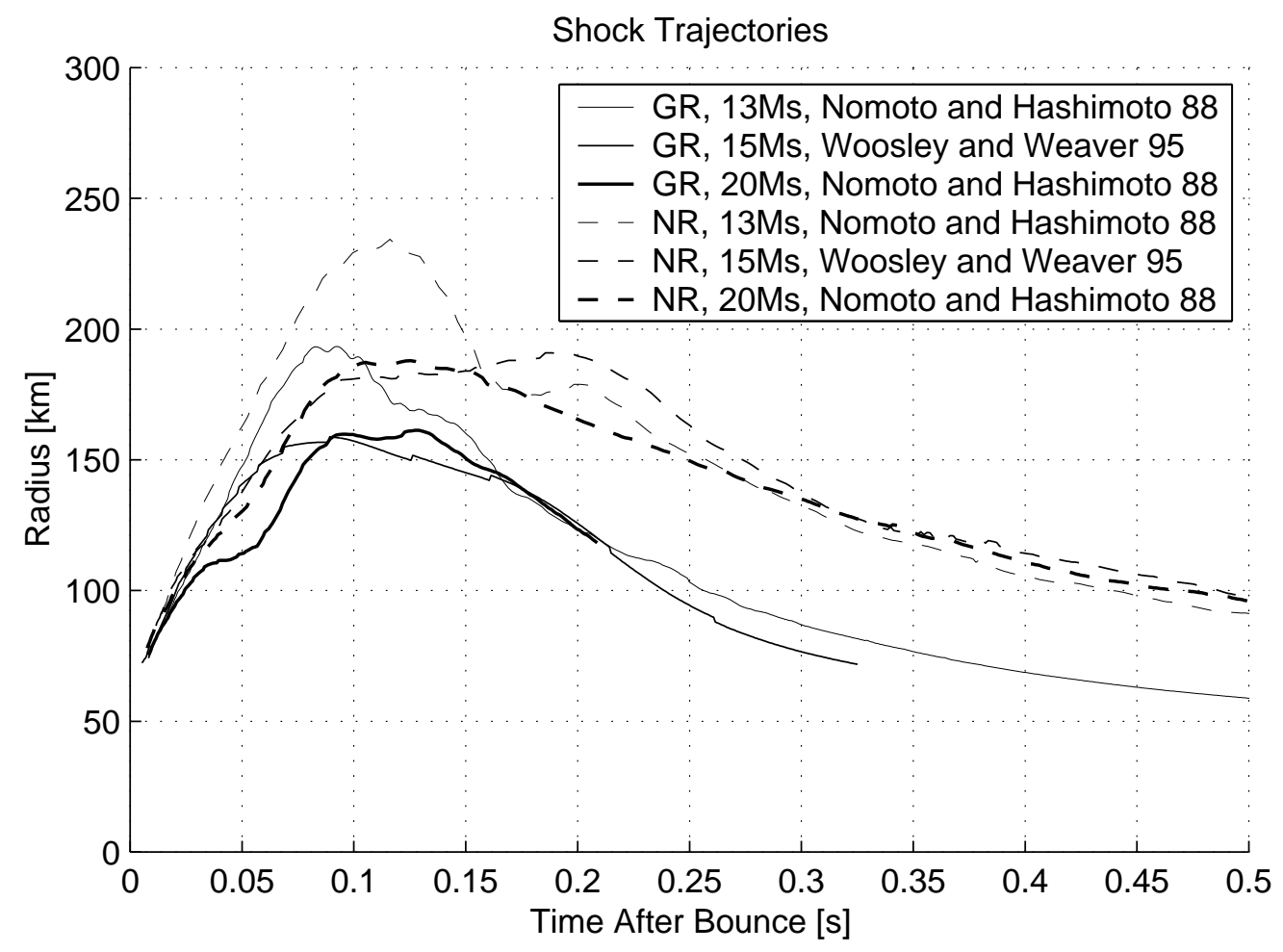

FIGURE 3. Shock trajectories for the GR and NR cases.

in the more compact GR case encounter material that has traversed a larger potential difference, producing more energetic accretion luminosities. After the shock has stalled, the surrounding layers (cooling/heating region, shock radius) adjust to a smaller radius and settle to a stationary equilibrium in the spirit of Burrows and Goshy [32]. In the GR simulation, this occurs at a smaller radius, deeper in the gravitational well, with higher infall velocities, leading to higher accretion luminosities with higher rms energies and higher heating rates (see also Bruenn et al. [23]).

This accretion-determined picture obtains additional support from the following observation: In the last graph in Fig. (1) we provide the mass flux at the electron neutrinosphere multiplied by the local gravitational potential. We can compare this energy rate to the luminosities of the electron flavor neutrinos that are shown in the second graph in Fig. (1). The striking similarity suggests that the luminosity is indeed mainly determined by the gravitational potential at the boundary of the proto-neutron star and the mass accretion rate. Moreover, we can directly relate the different accretion rates to differences in the density profiles of the initial progenitors. The energy accumulation rate at the neutrinospheres for the $15 \mathrm{M}_{\odot}$ model exceeds that of the $20 \mathrm{M}_{\odot}$ model between $60 \mathrm{~ms}$ and $160 \mathrm{~ms}$ after bounce. We have marked in Fig. (2) the region that crosses the neutrinospheres during this time. The density of the $15 \mathrm{M}_{\odot}$ progenitor exceeds the density of the $20 \mathrm{M}_{\odot}$ progenitor in exactly this region. Thus, the time dependent electron flavor luminosities reflect the position of the neutrinospheres and are directly modulated by the variations in the spatial density profiles of the progenitors. 


\section{ACKNOWLEDGMENTS}

M.L. is supported by the National Science Foundation under contract AST-9877130 and, formerly, was supported by the Swiss National Science Foundation under contract 2053798.98. O.E.M. is supported by funds from the Joint Institute for Heavy Ion Research and a Dept. of Energy PECASE award. A.M. is supported at the Oak Ridge National Laboratory, managed by UT-Battelle, LLC, for the U.S. Department of Energy under contract DE-AC05-00OR22725. W.R.H. is supported by NASA under contract NAG58405 and by funds from the Joint Institute for Heavy Ion Research. Our simulations were carried out on the ORNL Physics Division Cray J90 and the National Energy Research Supercomputer Center Cray J90.

\section{REFERENCES}

1. J. Tueller, S. Barthelmy, N. Gehrels, M. Leventhal, C. J. MacCallum, and B. J. Teegarden, in Supernovae, edited by S. E. Woosley, 278 (Berlin, Springer, 1991).

2. R. Strom., H. M. Johnston, F. Verbunt, and B. Aschenbach, Nature 373, 587 (1995).

3. T. J. Galama et al., Nature 395, 670 (1998).

4. D. C. Leonard, A. V. Filippenko, A. J. Barth, and T. Matheson, Astrophys. J. 536, 239 (2000).

5. M. Herant, W. Benz, and S. A. Colgate, Astrophys. J. 395, 642 (1992).

6. D. S. Miller, J. R. Wilson, and R. W. Mayle, Astrophys. J. 415, 278 (1993).

7. M. Herant, W. Benz, R. W. Hix, C. L. Fryer, and S. A. Colgate, Astrophys. J. 435, 339 (1994).

8. A. Burrows, J. Hayes, and B. A. Fryxell, Astrophys. J. 450, 830 (1995).

9. H.-T. Janka and E. Müller, Astronomy and Astrophysics 306, 167 (1996).

10. A. Mezzacappa, A. C. Calder, S. W. Bruenn, J. M. Blondin, M. W. Guidry, M. R. Strayer, and A. S. Umar, Astrophys. J. 495, 911 (1998).

11. C. F. Fryer, Astrophys. J. 522, 413 (1999).

12. C. F. Fryer and A. Heger, Astrophys. J. 541, 1033 (2000).

13. P. Höflich, J. C. Wheeler, L. Wang, Astrophys. J. 521, 179 (1999).

14. A. M. Khokhlov, P. A. Höflich, E. S. Oran, J. C. Wheeler, L. Wang, A. Yu. Chtchelkanova, Astrophys. J. Lett. 524, 107 (1999).

15. A. I. MacFadyen and S. E. Woosley, Astrophys. J. 524, 262 (1999).

16. J. C. Wheeler, I. Yi, P. Höflich, L. Wang, Astrophys. J. 537, 810 (2000).

17. A. Mezzacappa, M. Liebendörfer, O. E. B. Messer, R. W. Hix, F.-K. Thielemann, and S. W. Bruenn, accepted for publication in Phys. Rev. Lett., astro-ph/0005366 (2001).

18. A. Mezzacappa and S. W. Bruenn, Astrophys. J. 405, 669 (1993).

19. A. Mezzacappa and S. W. Bruenn, Astrophys. J. 405, 637 (1993).

20. A. Mezzacappa and S. W. Bruenn, Astrophys. J. 410, 740 (1993).

21. M. Liebendörfer, Ph.D. thesis, University of Basel, (Basel, Switzerland, 2000).

22. O. E. B. Messer, Ph.D. thesis, University of Tennessee, (Knoxville, USA, 2000).

23. S. W. Bruenn, K. R. DeNisco, and A. Mezzacappa, accepted for publication in Astrophys. J., astro$\mathrm{ph} / 0101400(2001)$.

24. M. Rampp and H.-T. Janka, Astrophys. J. Lett. 539, L33 (2000).

25. M. Liebendörfer, A. Mezzacappa, F.-K. Thielemann, O. E. B. Messer, R. W. Hix, S. W. Bruenn, submitted to Phys. Rev. D, astro-ph/0006418, (2001).

26. J. R. Wilson and R. W. Mayle, Physics Reports 227, 97 (1993).

27. K. Nomoto and M. Hashimoto, Physics Reports 163, 13 (1988).

28. S. E. Woosley and T. A. Weaver, Astrophys. J. Supplement 101, 181 (1995).

29. J. Lattimer and F. D. Swesty, Nuclear Physics A535, 331 (1991).

30. S. W. Bruenn, Astrophys. J. Supplement Series 58, 771 (1985).

31. H.-T. Janka, accepted for publication in Astronomy and Astrophysics, astro-ph/0008432 (2000).

32. A. Burrows and J. Goshy, Astrophys. J. Lett. 416, L75 (1993). 